\title{
CORRELAÇÃO ENTRE COMPONENTES DA PRODUTIVIDADE DA CANA-DE-AÇÚCAR COM pH DE UM ARGISSOLO VERMELHO DISTRÓFICO DO NOROESTE PAULISTA
}

\author{
César Gustavo da Rocha Lima ${ }^{1}$, Morel de Passos e Carvalho ${ }^{2}$, Adriano Souza $^{3}$, Nídia Raquel Costa ${ }^{4}$, Rafael \\ Montanari ${ }^{5}$
}

RESUMO

A produtividade agrícola depende primordialmente da capacidade produtiva dos solos. Assim, as práticas de adequação química do solo objetivam modificações nas suas características, com vistas a proporcionar melhores condições ao desenvolvimento das culturas. O presente estudo avaliou as correlações lineares e espaciais entre os atributos da produtividade da planta com o pH do solo. Na Fazenda Valença-I, Suzanápolis-SP, instalou-se uma malha geoestatística contendo 118 pontos amostrais, em uma área de 10,5 ha, onde foram analisados os atributos da canade-açúcar: produtividade de tonelada de colmos por hectare $(\mathrm{TCH})$, população de plantas (POP) e açúcares totais recuperáveis (ATR); e como atributos químicos do solo: o $\mathrm{pH}(\mathrm{pH})$ e Delta $\mathrm{pH}(\mathrm{DpH})$, em diferentes profundidades no perfil $(0-0,20 ; 0,20-0,40 ; 0,80-1,00 \mathrm{~m})$. Os resultados indicaram que a correlação linear entre os atributos da planta com os do solo é significativa. A melhor correlação ocorre entre TCH com o pH na profundidade de 0,80-1,00 m. Espacialmente, há uma apreciável resposta entre a produtividade com o $\mathrm{pH}$ do solo na camada de $0,20-0,40 \mathrm{~m}$. O DpH na camada de 0,80-1,00 m também apresenta importante interação com o ATR, o que permite inferir que melhor qualidade química na camada mais profunda proporciona melhor qualidade tecnológica do produto industrializável. Por fim, o estudo do solo nas camadas mais profundas (0,20-0,40 m e 0,80-1,00 m) aponta interação (linear e espacial) tanto com a produtividade quanto com o atributo tecnológico da cultura, apresentando-se como satisfatórios alvos de adequação química do solo.

Palavras-chave: agricultura de precisão, atributos químicos do solo, manejo do solo

\section{ABSTRACT \\ CORRELATION BETWEEN THE SUGARCANE PRODUCTIVITY WITH THE PH OF A RED ULTISOL DYSTROPHIC OF NORTHWEST OF SÃO PAULO STATE, BRAZIL}

Agricultural productivity depends primarily on the productive capacity of soils. Thus, practices of chemical suitability of the soil aim to changes in their characteristics to provide better conditions for the development of cultures. The present study aimed to evaluate the linear and spatial correlations between the attributes of plant productivity with soil $\mathrm{pH}$. The aim of this study was to evaluate the linear and spatial correlations between the attributes of the plant with soil. In Farm Valencia-I, Suzanápolis-SP, Brazil, a geostatistical grid with 118 sampling points has been installed in an area of 10.5 ha. The attributes of sugarcane analyzed was: productivity of tons of stems per hectare (TSH), plant population (PP) and total recoverable sugars (TRS); and as soil chemical properties: $\mathrm{pH}(\mathrm{pH})$ and Delta $\mathrm{pH}(\mathrm{DpH})$ at different depths $(0-0.20 ; 0.20-0.40 ; 0.80-1.00 \mathrm{~m})$. The results indicate that the linear correlation between the attributes of the soil with the plant is significant. The best correlation is between TRS with the $\mathrm{pH}$ in the depth of $0.80-1.00$ $\mathrm{m}$. Spatially, there is a significant interaction between productivity with the $\mathrm{pH}$ of the soil at a depth of $0.20-0.40 \mathrm{~m}$. The DpH depth of 0.80-1.00 m also correlates with the TRS, which allows inferring that best chemical quality in the deepest layer provides better technological quality of industrializable product. The soil study in the deeper layers (0.20-0.40 $\mathrm{m}$ and $0.80-1.00 \mathrm{~m})$ indicates interaction (linear and spatial) both productivity and with the technological attribute of sugarcane, presenting as target satisfactory of soil chemical suitability.

Keywords: precision agriculture, soil chemical attributes, soil management

Recebido para publicação em 25/02/2015. Aprovado em 11/04/2016.

1 - Geógrafo, Professor da Faculdade de Engenharia/UNESP, Ilha Solteira, SP, Brasil, cesarlima@dec.unesp.br

2 - Eng ${ }^{\circ}$. Agrônomo, Professor da Faculdade de Engenharia/UNESP, Ilha Solteira, SP, Brasil, mpcarva@agr.feis.unesp.br

3 - Eng. ${ }^{\circ}$ Civil, Professor da Faculdade de Engenharia/UNESP, Ilha Solteira, SP, Brasil, adriano@dec.feis.unesp.br

4 -Enga. Agrônoma, PPG - Faculdade de Ciências Agrárias/UNESP, Botucatu, SP, Brasil, nidiar costa@hotmail.com

5 - Eng ${ }^{\circ}$. Agrônomo, Professor da Faculdade de Engenharia/UNESP, Ilha Solteira, SP, Brasil, montanari@agr.feis.unesp.br

120 REVENG

120-130p.

ENGENHARIA NA AGRICULTURA, VIÇOSA - MG, V.24 N.2, MARÇO / ABRIL 2016 


\section{INTRODUÇÃO}

A cana-de-açúcar (Saccharum spp.) é uma gramínea de grande importância econômica para o Brasil e, apesar de sua múltipla utilidade, tem sido intensamente empregada como matéria prima pelo setor agroindustrial (FAHL et al., 1998). O processo industrial canavieiro visa principalmente produtividade, qualidade do produto industrializável, e a longevidade do canavial. Assim, sob tais perspectivas, o setor sucroalcooleiro do Brasil tem se destacado como o maior produtor mundial da cana-de-açúcar, apresentando uma produtividade média em torno de 74,7 t ha- ${ }^{-1}$ (CONAB, 2014).

$\mathrm{O}$ pH do solo é um dos atributos de maior influência no desenvolvimento das plantas, uma vez que está diretamente ligado à solubilidade, concentração, disponibilidade e capacidade de retenção da maioria dos nutrientes essenciais às culturas. De acordo com Raij (1991), o pH isoladamente possibilita ampla inferência sobre possíveis problemas com a fertilidade do solo ou mesmo com toxicidade, tornando-o um eficiente indicador qualitativo, pois a literatura tem evidenciando grande interação, entre causa efeito, deste atributo do solo com o desenvolvimento das culturas (SOUZA et al., 2007). Já o Delta pH indica o estado da carga elétrica no solo. Assim, quando o Delta $\mathrm{pH}$ é negativo indica que o solo possui uma maior CTC (VIEIRA, 1997), ao passo que o Delta pH positivo indicará maior CTA, retendo mais ânions.

A geoestatística tem sido amplamente utilizada e difundida como ferramenta na pesquisa agronômica, contribuindo à estatística clássica para o entendimento das diferentes interações espaciais entre os atributos químicos do solo e o desenvolvimento das culturas (CORÁ; BERALDO, 2006). O uso desta técnica de análise tem possibilitado a adequação pontual de determinadas áreas agrícolas proporcionando economia de insumos, aumento de produtividade e melhor qualidade dos produtos agrícolas. Assim, no Brasil, tem-se observado crescente aplicação da geoestatística nos estudos de manejo do solo voltado à produção da cana-de-açúcar, conforme observado nos trabalhos de Corá et al. (2004),
Marques Júnior et al. (2008), Mello et al. (2006), Souza et al. (2004) e Souza et al. (2007).

Diante do exposto, pressupondo que a variabilidade do $\mathrm{pH}$ do solo em maiores profundidades no perfil também exerça influência no desenvolvimento das culturas, objetivou-se analisar as correlações lineares e espaciais entre os atributos da produtividade e tecnológicos da canade-açúcar com o pH e Delta $\mathrm{pH}$ do solo pesquisado sob diferentes metodologias de avaliação em três camadas no perfil.

\section{MATERIAL E MÉTODOS}

A pesquisa foi realizada na Fazenda Valença I, no município de Suzanápolis, noroeste do Estado de São Paulo, Brasil, nas coordenadas 20²7'33"S e $51^{\circ} 08^{\prime} 05^{\prime}$ 'W. O clima é o Aw, segundo classificação de Köeppen, caracterizado como tropical úmido com estação chuvosa no verão e seca no inverno, com precipitação e temperaturas médias anuais de $1400 \mathrm{~mm}$ e $24,5{ }^{\circ} \mathrm{C}$, respectivamente. $\mathrm{O}$ solo, classificado de acordo com Embrapa (2013), foi um ARGISSOLO VERMELHO distrófico abrúptico textura média/argilosa, A moderado, hiperdistrófico, meso-epieutrófico, endoálico, endodênsico, epicompactado, muito profundo, fortemente ácido.

A cultura estudada foi a cana-de-açúcar (Saccharum spp.), variedade RB 855453, implantada sobre área de pastagem com Brachiaria decumbens. A dessecagem foi feita com glifosato $\left(1,8 \mathrm{~kg}\right.$ ia ha $\left.{ }^{-1}\right)$. No preparo do solo, foram realizadas uma gradagem pesada (SGAC 18) e duas gradagens intermediárias (SIC 28) e a aplicação a lanço de 2,0 t ha ${ }^{-1}$ de calcário dolomítico, sendo utilizado para sua incorporação, a $0,35 \mathrm{~m}$, um arado de aivecas.

O plantio foi realizado em abril de 2010, utilizando-se um sulcador de duas linhas $(1,50 \mathrm{~m})$. No momento da realização do sulco utilizou-se $500 \mathrm{~kg} \mathrm{ha}^{-1}$ da fórmula 6-30-24 e para cobrição dos toletes foi aplicado fipronil (200 $\mathrm{g}$ ia ha $\left.{ }^{-1}\right)$. Após o primeiro corte (mecanizado), foi feita aplicação de $1 \mathrm{t} \mathrm{ha}^{-1}$ de gesso e $500 \mathrm{~kg} \mathrm{ha}^{-1}$ da fórmula 18-0027. Após o segundo corte, realizou-se adubação da soqueira com $400 \mathrm{~kg} \mathrm{ha}^{-1} \mathrm{da}$ fórmula 18-00-27. Na ocasião da coleta de dados, a cultura encontrava-se no terceiro corte e bisada. 
Para coleta dos dados, foi alocada uma malha geoestatística amostral constituída de 118 pontos, distribuídos aleatoriamente de forma a cobrir toda a área do talhão. O perímetro de abrangência dos pontos de coleta cobriu uma extensão de aproximadamente 10,5 ha. Na malha de coleta os pontos amostrais espaçavam-se, na grande malha, em aproximadamente $34 \mathrm{~m}$, ao passo que na malha de refinamento em aproximadamente $17 \mathrm{~m}$.

Foram determinados os atributos químicos do solo e da planta, individualmente coletados no entorno de cada ponto amostral. Para a coleta dos atributos da planta, definiu-se uma área útil, compreendendo duas linhas de $3 \mathrm{~m}$ de cultivo $\left(9 \mathrm{~m}^{2}\right)$ ao redor de cada ponto amostral. Para determinação da produtividade da cana-deaçúcar (Tonelada de Colmos por Hectares -TCH), utilizou-se uma balança eletrônica portátil. Para obtenção do peso por ponto, os colmos da área útil foram cortados simulando a colheita manual sendo, posteriormente, organizados em feixes, pesados e somados. Os dados de pesagem de THC coletados em campo, foram transferidas para uma planilha do Excel, na qual foi realizada a extrapolação dos valores da área útil de $9 \mathrm{~m}^{2}$ para 1 ha. Na obtenção da População de Plantas (POP), expresso em plantas $\mathrm{m}^{-2}$, utilizou-se uma trena para marcar $3 \mathrm{~m}$ lineares e, assim, executou-se a contagem do número de plantas industrialmente viáveis por ponto. Na determinação dos Açúcares Totais Recuperáveis (ATR), foram coletados em sequência na touceira 10 colmos industrializáveis. Os feixes identificados foram despontados e avaliados em laboratório, onde obteve-se o ATR, em kg t ${ }^{-1}$ de colmos (CONSECANA, 2006).

Em amostras coletadas na entrelinha da semeadura da cana-de-açúcar, nas profundidades de $0-0,20 \mathrm{~m} ; 0,20-0,40 \mathrm{~m}$ e $0,80-1,00 \mathrm{~m}$, utilizando para tal um trado caneca, foram medidos o pH em água (pHa), pH em cloreto de cálcio $(\mathrm{pHc})$ e o $\mathrm{pH}$ em cloreto de potássio (pHk). As amostras deformadas do solo foram secas ao ar (TFSA) e posteriormente passadas por uma peneira de $2,0 \mathrm{~mm}$. O pHa foi determinado por meio de eletrodo combinado imerso em suspensão solo/ água $(1: 2,5)$. O pHc foi determinado em solução de $\mathrm{CaCl}_{2}$ 0,025 $\mathrm{mol} \mathrm{L}^{-1}(1: 2,5)$ ao passo que o $\mathrm{pHk}$ foi determinado em solução de $\mathrm{KCl} 1 \mathrm{~mol} \mathrm{~L}^{-1}(1: 2,5)$, conforme Embrapa (2009). O Delta pH (DpH) foi calculado como sendo a diferença entre o valor do $\mathrm{pH}$ determinado em cloreto de potássio e o $\mathrm{pH}$ determinado em água.

Para cada atributo foi efetuada a análise descritiva clássica com o auxílio do software estatístico SAS (SCHLOTZHAVER; LITTELL, 1997). Na avaliação da normalidade, ou de lognormalidade, para cada atributo, realizou-se o teste de Shapiro e Wilk a $1 \%$ de probabilidade. Com o auxílio da planilha de cálculos Excel, montou-se a matriz de correlação objetivando efetuar as correlações lineares simples entre as combinações, duas a duas, de todos os atributos para posteriormente modelar as regressões de interesse agronômico.

A análise espacial foi realizada pelo uso do pacote geoestatístico Gamma Design Software 7.0 - GS+ (ROBERTSON, 2004). Assim, isoladamente para cada atributo, foi analisada sua dependência espacial pelo cálculo do semivariograma simples. Aos atributos que apresentaram tendência em seus dados foi aplicada a técnica do refinamento polinomial (DALCHIAVON et al., 2011). Seguidamente com o uso do software GS+, ajustaram-se os semivariogramas cruzados entre as combinações de atributos da planta vs. solo.

Os ajustes semivariográficos, simples e cruzados, foram efetuados pela seleção inicial de: a) a menor soma dos quadrados dos desvios (RSS); b) o maior coeficiente de determinação $\left(\mathrm{r}^{2}\right)$, e c) o maior avaliador da dependência espacial (ADE). A análise do ADE foi efetuada conforme expressão contida em Robertson (2004):

$\mathrm{ADE}=\left[\mathrm{C} /\left(\mathrm{C}+\mathrm{C}_{0}\right)\right] .100$

em que,

$\mathrm{ADE}=$ avaliador da dependência espacial;

$\mathrm{C}=$ variância estrutural; e

$\mathrm{C}+\mathrm{C}_{0}=$ o patamar.

A interpretação proposta para o ADE foi a seguinte: a) $\mathrm{ADE} \leq 25 \%$ indicando variável espacial fracamente dependente; b) $25 \%<\mathrm{ADE} \leq$ $75 \%$ moderadamente dependente, e c) ADE $>75 \%$ fortemente dependente. 


\section{RESULTADOS E DISCUSSÃO}

No Quadro 1 está apresentado os resultados da análise estatística descritiva dos atributos da produtividade da cana-de-açúcar e dos atributos químicos do solo avaliados.

De Acordo com Freddi et al. (2006), a variabilidade de um atributo pode ser classificada conforme a magnitude do seu coeficiente de variação. Assim, na análise descritiva (Quadro 1), para o atributo da planta $(\mathrm{TCH})$ foi observado alta variabilidade dos dados $(\mathrm{CV}=23,0 \%)$ similares ao trabalho de Souza et al. (2008) no qual apontaram $\mathrm{CV}$ da mesma magnitude. Do mesmo modo, a população de plantas (POP) também apresentou alta variabilidade $(\mathrm{CV}=20,9 \%)$. Já o ATR apresentou média variabilidade $(\mathrm{CV}=11,9 \%)$.

$\mathrm{O}$ pH do solo (Quadro 1), nas camadas de 0 - 0,20 $\mathrm{m}$ e $0,20-0,40 \mathrm{~m}$, apresentou baixa variabilidade dos dados indiferente da metodologia utilizada. Já na camada mais profunda $(0,80-1,00 \mathrm{~m})$ o $\mathrm{pHa}$ apresentou $\mathrm{CV}$ baixo, ao passo que o $\mathrm{pHk}$ e $\mathrm{pHc}$ apresentaram médio $\mathrm{CV}$. Tal diferença entre suas variabilidades pode ter ocorrido devido à possível presença de sais solúveis na camada mais profunda do solo, influenciando a força iônica da solução na determinação do $\mathrm{pH}$ em água. Fato esse que não ocorre quando o $\mathrm{pH}$ é determinado em solução de $\mathrm{KCl}$ e $\mathrm{CaCl}_{2}$ (NOVAIS et al., 2007). Por outro lado, essa diferença observada entre os atributos do $\mathrm{pH}$ ficou em sintonia com os trabalhos de Campos et al. (2007) Corá et al. (2004), Mello et al. (2006)

Quadro 1. Análise descritiva dos atributos da produtividade da cana-de-açúcar e do pH de um Argissolo Vermelho distrófico.

\begin{tabular}{|c|c|c|c|c|c|c|c|c|c|}
\hline \multirow{3}{*}{ Atributo $^{(a)}$} & \multicolumn{9}{|c|}{ Medidas estatísticas descritivas } \\
\hline & \multirow{2}{*}{ Média } & \multicolumn{2}{|c|}{ Valor } & \multirow{2}{*}{$\begin{array}{l}\text { Desvio } \\
\text { Padrão }\end{array}$} & \multicolumn{3}{|c|}{ Coeficiente } & \multicolumn{2}{|c|}{ Probabilidade $^{(\mathrm{b})}$} \\
\hline & & Mínimo & Máximo & & Variação (\%) & Curtose & Assimetria & $\operatorname{Pr}<\mathbf{w}$ & DF \\
\hline \multicolumn{10}{|c|}{ Atributos da Planta } \\
\hline$T C H\left(t h a^{-1}\right)$ & 89,51 & 42,00 & 153,01 & 20,618 & 23,0 & 0,075 & 0,143 & 0,3860 & NO \\
\hline $\operatorname{ATR}\left(\mathrm{kg} \mathrm{t}^{-1}\right)$ & 136,0 & 90,69 & 171,84 & 16,179 & 11,9 & 0,209 & $-0,466$ & 0,0708 & NO \\
\hline$P O P\left(p l m^{-2}\right)$ & 6,55 & 2,44 & 10,67 & 1,374 & 20,9 & 0,763 & 0,047 & 0,4665 & NO \\
\hline \multicolumn{10}{|c|}{ Atributos do Solo $(0-0,20 \mathrm{~m})$} \\
\hline pHa1 & 5,87 & 5,30 & 6,60 & 0,247 & 4,2 & $-0,013$ & 0,122 & 0,0313 & $\mathrm{TL}$ \\
\hline pHk1 & 4,83 & 4,30 & 5,50 & 0,257 & 5,3 & $-0,479$ & 0,055 & 0,0402 & $\mathrm{TN}$ \\
\hline pHc1 & 4,64 & 4,00 & 5,20 & 0,233 & 5,0 & $-0,326$ & $-0,016$ & 0,0656 & NO \\
\hline $\mathrm{DpHI}$ & $-1,05$ & $-1,40$ & $-0,70$ & 0,160 & 15,3 & $-0,166$ & $-0,353$ & 0,0001 & IN \\
\hline \multicolumn{10}{|c|}{ Atributos do Solo $(0,20-0,40 \mathrm{~m})$} \\
\hline pHa2 & 6,01 & 5,40 & 6,70 & 0,240 & 4,0 & $-0,100$ & 0,083 & 0,0561 & NO \\
\hline pHk2 & 4,97 & 4,40 & 5,70 & 0.282 & 5,7 & $-0,396$ & 0,023 & 0,0441 & $\mathrm{TL}$ \\
\hline pHc2 & 4,75 & 4,30 & 5,40 & 0,249 & 5,2 & $-0,479$ & 0,199 & 0,0204 & $\mathrm{TL}$ \\
\hline $\mathrm{DpH} 2$ & $-1,04$ & $-1,50$ & $-0,500$ & 0,163 & 15,7 & 0,548 & $-0,205$ & 0,0003 & IN \\
\hline \multicolumn{10}{|c|}{ Atributos do Solo $(0,80-1,00 \mathrm{~m})$} \\
\hline pHa3 & 5,99 & 5,20 & 6,80 & 0,364 & 6,1 & $-0,767$ & $-0,107$ & 0,0123 & IN \\
\hline pHk3 & 4,94 & 4,30 & 5,90 & 0,527 & 10,7 & $-1,456$ & 0,310 & 0,0001 & IN \\
\hline pHc3 & 4,78 & 4,20 & 5,90 & 0,486 & 10,2 & $-1,072$ & 0,523 & 0,0001 & IN \\
\hline DpH3 & $-1,06$ & $-1,90$ & $-0,40$ & 0,292 & 27,5 & 0,301 & $-0,365$ & 0,0507 & LN \\
\hline
\end{tabular}

(a) TCH refere-se à produtividade de colmos por hectare; POP refere-se à população de plantas por $\mathrm{m}^{-2}$; ATR refere-se aos açúcares totais recuperáveis; $\mathrm{pHa}, \mathrm{pHk}, \mathrm{pHc}$ e $\mathrm{DpH}$ são respectivamente atributos da reação do solo nas respectivas camadas (1: 0 - 0,20 m; 2: 0,20 - 0,40 m; 3: 0,80 - 1,00 m); (b) DF = distribuição de frequência, sendo NO, TN, LN, TL e IN respectivamente do tipo normal, tendendo à normal, lognormal, tendendo à lognormal e indefinido. 
e Souza et al. (2007; 2010), nos quais também foram encontrados dados apresentando ora baixa ora média variabilidade. Já o Delta $\mathrm{pH}$ apresentou média variabilidade nas camadas de $0-0,20 \mathrm{~m}$ e $0,20-0,40 \mathrm{~m}$ e alta variabilidade na camada de $0,80-1,00 \mathrm{~m}$.

No Quadro 1, apresenta-se o valor médio para produtividade de colmos $(\mathrm{TCH})(89,5 \mathrm{t}$ ha$\left.{ }^{1}\right)$, que está acima da média brasileira na safra 2013/2014 (74,7 $\left.\mathrm{t} \mathrm{ha}^{-1}\right)$ (CONAB, 2014). Por outro lado, por se tratar de cana bisada, deduz-se que o rendimento não foi tão satisfatório, principalmente pelo fato de Fahl et al. (1998) ter apontado para o Estado de São Paulo produtividades em torno de $85 \mathrm{t} \mathrm{ha}^{-1}$, no quinto corte. Sabe-se que a baixa população de plantas pode influenciar diretamente a produtividade das culturas. Assim, no presente trabalho, a POP apresentou valor médio de 6,5 plantas $\mathrm{m}^{-2}$, considerado abaixo do ideal para o espaçamento utilizado $(1,5 \mathrm{~m})$, que seria um valor médio de $10,5 \mathrm{pl} . \mathrm{m}^{-2}$. Deste modo, a baixa população de plantas observada (Quadro 1) deve ter corroborado para que a produção da cana estivesse abaixo do esperado, uma vez que, para cana bisada no terceiro corte, esperava-se um maior rendimento de fitomassa/área. Já com relação ao ATR, o seu valor médio $\left(136 \mathrm{~kg} \mathrm{t}^{-1}\right)$ está acima dos valores considerados como padrão (em torno de $122 \mathrm{~kg} \mathrm{t}^{-1}$ ) conforme apresentado por Segato et al. (2006).

Os dados observados aos atributos do solo (Quadro 1) indicaram que os valores de $\mathrm{pH}$ foram mais elevados para $\mathrm{pH}$ em água ( $\mathrm{pHa}$ ) em todas as camadas. Tal fato deve-se principalmente a influência de sais no solo (RAIJ, 1991; NOVAIS et al., 2007). Ainda com base nos dados apresentados no Quadro 1, pode-se inferir que o $\mathrm{pH}$ do solo avaliado $\left(\mathrm{KCl}\right.$ e $\left.\mathrm{CaCl}_{2}\right)$, em todas as camadas do solo, apresentou valores abaixo da média (entre 5,5 e 6,5 ) considerada ideal para disponibilidade da maioria dos nutrientes, indicando assim, elevada acidez conforme Raij et al. (1997). Entre as camadas estudadas $(0-0,20 ; 0,20-0,40$ e 0,80 - 1,00 m) observou-se que a diferença nos valores de $\mathrm{pH}$ foi pequena, porém indicou um aumento gradual em profundidade no perfil, provavelmente devido a calagem e gessagem realizadas no momento do preparo do solo, associado ao processo natural de percolação em profundidade no perfil.
De modo geral, as operações de preparo do solo proporcionaram um ambiente mais homogêneo também em profundidade no perfil. Do mesmo modo, o DpH médio apresentou-se bem uniforme no perfil, com valores negativos indicando maior capacidade de retenção de cátions em todas as camadas.

Das correlações avaliadas entre os atributos da planta (Quadro 2), houve significância entre os pares TCH vs POP $\left(r=0,388^{* *}\right)$ e ATR vs POP $(r=$ $\left.-0,178^{*}\right)$. A primeira correlação de maior interesse prático agronômico evidenciou correlação positiva e altamente significativa com TCH. Assim, em relação à $\mathrm{TCH}$, a POP foi um atributo de incremento direto da produção, algo esperado, uma vez que o número de indivíduos tem sido fator determinante para definir a melhor produtividade da cultura.

Das correlações entre os atributos da planta vs. solo (Quadro 2), observou-se significância exclusiva entre os pares TCH vs. pHc2 $(\mathrm{r}=$ $\left.0,183^{*}\right), \mathrm{TCH}$ vs. $\mathrm{DpH} 2\left(\mathrm{r}=0,228^{*}\right)$, TCH $v s$. pHa3 $\left(\mathrm{r}=0,218^{*}\right)$, TCH $v s . \mathrm{pHk} 3\left(\mathrm{r}=0,299^{* *}\right)$, TCH vs. pHc3 $\left(\mathrm{r}=0,294^{* *}\right)$ e, TCH $v$. DpH3 $(\mathrm{r}=$ $\left.0,268^{* *}\right)$, todos de forma direta. Deste modo, com o aumento do $\mathrm{pH}$, principalmente na camada mais profunda, haverá o aumento na produtividade, seja pelo aumento da disponibilidade de nutrientes, ou pela redução de possíveis efeitos de toxicidade.

Conforme observado neste estudo (Quadro 2), algumas pesquisas têm indicado que, apesar de significativas, tem sido baixas as correlações lineares entre atributos químicos do solo com a produtividade das culturas (Souza et al., 2008). Assim, em virtude de baixos coeficientes de correlação observado entre os atributos da planta vs. solo, Souza et al. (2008) com base em seu trabalho e demais referências (Souza et al., 2004) relatou que o relevo tem sido um atributo de definição da distribuição de atributos químicos do solo, apresentando altas correlações com a produtividade de culturas. Porém, do ponto de vista prático, o relevo não é comumente passível de manejo agrícola como os demais atributos químicos, principalmente por ele (relevo) agregar a influência de um conjunto de fatores, diferentemente da observação de um exclusivo atributo passível de adequação como o pH (calagem, gessagem). Portanto, diferentemente do relevo, os atributos 
Quadro 2. Matriz de correlação linear simples entre atributos da produtividade da cana-de-açúcar e do pH de um Argissolo Vermelho distrófico.

\begin{tabular}{|c|c|c|c|c|c|c|c|c|c|c|c|c|c|c|}
\hline Atributo $^{(a)}$ & $\begin{array}{c}\text { Coeficiente } \\
\text { de } \\
\text { correlação }^{(b)}\end{array}$ & & & & & & & & & & & & & \\
\hline & TCH & $P O P$ & $A T R$ & pHa1 & pHk1 & pHc1 & $D p H 1$ & $p H a 2$ & $p H k 2$ & $p H c 2$ & $\mathrm{DpH} 2$ & pHa3 & pHk3 & pHc3 \\
\hline POP & $0.388^{* *}$ & & & & & & & & & & & & & \\
\hline ATR & $-0.028^{\mathrm{ns}}$ & $-0.178^{*}$ & & & & & & & & & & & & \\
\hline pHal & $-0.020^{\mathrm{ns}}$ & $0.124^{\mathrm{ns}}$ & $-0.093^{\mathrm{ns}}$ & & & & & & & & & & & \\
\hline$p H k 1$ & $0.026^{\text {ns }}$ & $0.075^{\mathrm{ns}}$ & $-0.124^{\mathrm{ns}}$ & $0.777^{* *}$ & & & & & & & & & & \\
\hline pHc1 & $0.073^{\text {ns }}$ & $0.126^{\mathrm{ns}}$ & $-0.104^{\mathrm{ns}}$ & $0.718^{* *}$ & $0.899^{* *}$ & & & & & & & & & \\
\hline DpHI & $0.057^{\text {ns }}$ & $-0.083^{\mathrm{ns}}$ & $-0.067^{\mathrm{ns}}$ & $-0.255^{* *}$ & $0.385^{* *}$ & $0.326^{* *}$ & & & & & & & & \\
\hline pHa2 & $-0.005^{\mathrm{ns}}$ & $0.236^{*}$ & $-0.211^{*}$ & $0.750^{* *}$ & $0.643^{* *}$ & $0.686^{* *}$ & $-0.102^{* *}$ & & & & & & & \\
\hline$p H k 2$ & $0.128^{\text {ns }}$ & $0.229^{*}$ & $-0.217^{*}$ & $0.564^{* *}$ & $0.702^{* *}$ & $0.711^{* *}$ & $0.252^{* *}$ & $0.816^{* *}$ & & & & & & \\
\hline$p H c 2$ & $0.183^{*}$ & $0.233^{*}$ & $-0.155^{\mathrm{ns}}$ & $0.524^{* *}$ & $0.627^{* *}$ & $0.688^{* *}$ & $0.202^{*}$ & $0.791^{* *}$ & $0.927^{* *}$ & & & & & \\
\hline $\mathrm{DpH} 2$ & $0.228^{*}$ & $0.049^{\text {ns }}$ & $-0.065^{\mathrm{ns}}$ & $-0.127^{\mathrm{ns}}$ & $0.269^{* *}$ & $0.219^{*}$ & $0.585^{* *}$ & $-0.060^{\mathrm{ns}}$ & $0.528^{* *}$ & $0.438^{* *}$ & & & & \\
\hline pHa3 & $0.218^{*}$ & $0.223^{*}$ & $0.029^{\text {ns }}$ & $0.323^{* *}$ & $0.204^{*}$ & $0.289^{* *}$ & $-0.185^{*}$ & $0.410^{* *}$ & $0.336^{* *}$ & $0.394^{* *}$ & $-0.022^{\mathrm{ns}}$ & & & \\
\hline pHk3 & $0.299^{* * *}$ & $0.167^{\mathrm{ns}}$ & $0.070^{\mathrm{ns}}$ & $0.165^{\mathrm{ns}}$ & $0.077^{\mathrm{ns}}$ & $0.139^{\text {ns }}$ & $-0.158^{*}$ & $0.258^{* *}$ & $0.249^{* *}$ & $0.302^{* *}$ & $0.052^{\mathrm{ns}}$ & $0.848^{* * *}$ & & \\
\hline$p H c 3$ & $0.294^{* * *}$ & $0.205^{*}$ & $0.088^{\mathrm{ns}}$ & $0.218^{*}$ & $0.083^{\mathrm{ns}}$ & $0.155^{\mathrm{ns}}$ & $-0.225^{* *}$ & $0.291^{* *}$ & $0.242^{* *}$ & $0.299^{* *}$ & $-0.010^{\mathrm{ns}}$ & $0.845^{\text {*** }}$ & $0.980^{* *}$ & \\
\hline DpH3 & $0.268^{* *}$ & $0.024^{\mathrm{ns}}$ & $0.091^{\mathrm{ns}}$ & $-0.106^{\mathrm{ns}}$ & $-0.115^{\mathrm{ns}}$ & $-0.109^{\text {ns }}$ & $-0.054^{\mathrm{ns}}$ & $-0.046^{\mathrm{ns}}$ & $0.031^{\mathrm{ns}}$ & $0.054^{\mathrm{ns}}$ & $0.121^{\mathrm{ns}}$ & $0.285^{* * *}$ & $0.750^{* *}$ & $0.717^{7 *}$ \\
\hline
\end{tabular}

(a) TCH refere-se à produtividade de colmos por hectare; POP refere-se à população de plantas por $\mathrm{m}^{-2}$; ATR refere-se aos açúcares totais recuperáveis; $\mathrm{pHa}, \mathrm{pHk}, \mathrm{pHc}$ e $\mathrm{DpH}$ são respectivamente atributos da reação do solo nas respectivas camadas (1: 0 - 0,20 $\mathrm{m} ; 2: 0,20-0,40 \mathrm{~m} ; 3: 0,80-1,00 \mathrm{~m})$; (b) * significativo ao nível de 5\% de probabilidade; ** significativo ao nível de $1 \%$ de probabilidade; ${ }^{\text {ns }}$ não significativo.

químicos e físicos do solo, mesmo apresentando baixas correlações, quando significativas, devem ser tecnicamente consideradas.

Assim, a TCH pode ser estimada em função dos atributos que apresentaram as melhores correlações, sendo por meio da POP (como atributo da planta), ou por meio do $\mathrm{pH} 3$ e do $\mathrm{pHc} 3$ (como atributos do solo), a partir das seguintes equações de regressão:

$$
\begin{aligned}
& \mathrm{TCH}=5,518 \cdot 10^{1} \cdot \exp ^{\text {POP } \cdot 6,929 \cdot 10^{\wedge 2^{* *}}} \quad(\mathrm{r}=0,393, \mathrm{p}<0,01) \\
& \mathrm{TCH}=3,172 \cdot 10^{1}+1,169 \cdot 10^{1 * *} \cdot \mathrm{pHk} 3 \quad(\mathrm{r}=0,299, \mathrm{p}<0,01) \\
& \mathrm{TCH}=2,997 \cdot 10^{1}+1,245 \cdot 10^{1 * *} \cdot \mathrm{pHc} 3 \quad(\mathrm{r}=0,294, \mathrm{p}<0,01)
\end{aligned}
$$

Deste modo, a relação exponencial entre $\mathrm{TCH}$ vs POP, dada pelo modelo matemático descrito na equação 2, indicou que, onde a POP variou de 2,410,7 pl. $\mathrm{m}^{-2}$, a TCH apresentou valores da ordem de $65,1-115,5 \mathrm{t} \mathrm{ha}^{-1}$. Esse intervalo de dados infere que a população ideal, para uma produtividade máxima nas mesmas condições de campo, seria de 10,7 pl. $\mathrm{m}^{-2}$, equivalente a aproximadamente 16 plantas por metro.
Já com relação às regressões modeladas entre planta vs solo [equações (3) e (4)], estas combinações apresentaram interação direta de causa e efeito entre o atributo da planta com o $\mathrm{pH}$ do solo na camada de $0,80-1,00 \mathrm{~m}$. Indicando que a camada menos impactada pelo manejo agrícola possibilitou observar as melhores interações com $\mathrm{o}$ atributo da planta. Assim, a melhor relação linear dada entre TCH vs pHk3, [equação (3)], indicou que, o aumento de 1 décimo nos valores de $\mathrm{pHk} 3$ resultou, para a área estudada, um incremento de $1,17 \mathrm{t} \mathrm{ha}^{-1}$ na produtividade de colmos da canade-açúcar. Portanto, do ponto de vista prático, a qualidade química do solo na camada mais profunda se mostrou, no presente estudo, como um dos fatores de incremento da produtividade da cana-de-açúcar.

A análise espacial (Quadro 3) evidenciou, à exceção da POP e \#pHk3 que apresentaram efeito pepita puro (epp.), apreciáveis semivariogramas. Assim, a análise dos parâmetros semivariográficos simples revelou para produtividade da cana (\#TCH) ajuste do tipo exponencial, com alcance 
de $274,8 \mathrm{~m}$, coeficiente de determinação espacial $\left(\mathrm{r}^{2}\right)$ de 0,882 , e médio grau de dependência espacial (ADE). Outros estudos da dependência espacial da produtividade da cana-de-açúcar têm mostrado também apreciáveis ajustes semivariográficos como contido em Souza et al. (2010). O ATR apresentou ajuste do tipo esférico, com alcance de $243,4 \mathrm{~m}$, coeficiente de determinação espacial $\left(\mathrm{r}^{2}\right)$ de 0,904 , e médio ADE.

A análise dos parâmetros semivariográficos simples dos atributos químicos do solo (Quadro $3)$ revelou ajustes do tipo esférico (pHa1, pHk1), do tipo exponencial ( $\mathrm{pHc} 1, \mathrm{pHc} 2, \mathrm{pHa} 2, \mathrm{pHk} 2)$ e do tipo gaussiano ( $\mathrm{DpH} 1, \mathrm{DpH} 2, \mathrm{DpH} 3, \mathrm{\# pHa3}$, \#pHc3), com alcances variando de $75 \mathrm{~m}$ (pHc3) a 465,9 m (DpH1). No geral, observou-se apreciável desempenho dos atributos do solo dado pelo ADE que variou de 25,5\% (\#pHc3) a 75,52\% (pHc1).

À exceção do Delta $\mathrm{pH}(\mathrm{DpH})$, para o qual ainda se nota alguma dificuldade em encontrar trabalhos de referência na sua avaliação, o pH do solo avaliado apresentou, no geral, parâmetros semivariográficos em sintonia com os dados apresentados em diversos trabalhos (Berner et al., 2007; Campos et al., 2007; Corá et al., 2004; Mello et al., 2006) uma vez que neles, ora um ora outro modelo de ajuste (esférico, gaussiano e exponencial), foi observado, bem como alcances (de 8,5-783,0 m), e ADE (de moderado a alto). Assim, para a área em questão, nos trabalhos futuros de caráter semelhante, os valores dos alcances a serem utilizados nos pacotes geoestatísticos não deverão ser menores do que $75,0 \mathrm{~m}$.

No Quadro 4, estão apresentados os parâmetros dos semivariogramas cruzados ajustados entre os atributos da planta. Desta forma, a cokrigagem \#TCH=f(ATR) apresentou dependência espacial indireta entre causa e efeito, com ajuste gaussiano e alcance de $248,2 \mathrm{~m}$. Indicando uma relação antagônica entre produtividade e qualidade do produto industrializável. Assim, nas áreas de maiores valores de ATR, serão as áreas onde o TCH apresentará seus menores valores, e vice-versa.

Quadro 3. Parâmetros dos semivariogramas simples ajustados para os atributos da produtividade da canade-açúcar e do $\mathrm{pH}$ de um Argissolo Vermelho distrófico.

\begin{tabular}{|c|c|c|c|c|c|c|c|c|c|c|}
\hline \multirow{3}{*}{ Atributos $^{(a)}$} & \multicolumn{10}{|l|}{$\begin{array}{c}\text { Parâmetros } \\
\text { do ajuste }\end{array}$} \\
\hline & \multirow{2}{*}{ Modelo $^{(\mathbf{b})}$} & \multirow{2}{*}{$\mathrm{C}_{\mathrm{o}}$} & \multirow{2}{*}{$\mathrm{C}_{\mathrm{o}}+\mathrm{C}$} & \multirow{2}{*}{$\mathbf{A}_{0}(\mathbf{m})$} & \multirow{2}{*}{$\mathbf{r}^{2}$} & \multirow{2}{*}{$\mathbf{S Q R}^{(\mathrm{c})}$} & \multirow{2}{*}{$\begin{array}{c}\mathrm{ADE}^{(\mathrm{d})} \\
\% \\
\end{array}$} & \multicolumn{3}{|c|}{ validação cruzada } \\
\hline & & & & & & & & $\mathbf{a}$ & $\mathbf{B}$ & $\mathbf{R}$ \\
\hline \multicolumn{11}{|c|}{$\gamma(h)$ simples da planta } \\
\hline$T C H\left(t h a^{-1}\right)$ & $\exp$ & $1,739.10^{2}$ & $3,479.10^{2}$ & 274,8 & 0,882 & $1,850.10^{3}$ & 50,0 & $-3,78$ & 0,746 & 0,288 \\
\hline$P O P\left(p l m^{-2}\right)$ & epp & 1,950 & 1,950 & - & - & - & - & - & - & - \\
\hline $\operatorname{ATR}\left(k g t^{1}\right)$ & esf & $9,380.10^{1}$ & $2,112.10^{2}$ & 243,4 & 0,904 & $1,160.10^{3}$ & 55,6 & 7,41 & 0,946 & 0,546 \\
\hline \multicolumn{11}{|c|}{$\gamma(h)$ simples do solo $0-0,20 \mathrm{~m}$} \\
\hline pHa1 & esf & $3,410.10^{-2}$ & $6,510.10^{-2}$ & 184,2 & 0,860 & $7,521.10^{-5}$ & 47,6 & $3,90.10^{-1}$ & 0,934 & 0,499 \\
\hline pHk1 & esf & $3,515 \cdot 10^{-2}$ & $6,430 \cdot 10^{-2}$ & 179,0 & 0,983 & $6,687 \cdot 10^{-6}$ & 45,3 & $3,30.10^{-1}$ & 0,934 & 0,438 \\
\hline pHc1 & $\exp$ & $1,060.10^{-2}$ & $4,330 \cdot 10^{-2}$ & 100,5 & 0,959 & $5,657 \cdot 10^{-6}$ & 75,5 & 1,30 & 0,721 & 0,311 \\
\hline$D p H 1$ & gau & $1,805.10^{-2}$ & $3,553.10^{-2}$ & 416,5 & 0,981 & $6,880.10^{-6}$ & 49,2 & $-1,00.10^{-1}$ & 0,903 & 0,366 \\
\hline \multicolumn{11}{|c|}{$\gamma(h)$ simples do solo $0,20-0,40 \mathrm{~m}$} \\
\hline pHa2 & exp & $2,960.10^{-2}$ & $6,130 \cdot 10^{-2}$ & 324,0 & 0,980 & $9,702 \cdot 10^{-6}$ & 51,7 & $6,60.10^{-1}$ & 0,890 & 0,444 \\
\hline pHk2 & exp & $3,460.10^{-2}$ & $7,210.10^{-2}$ & 107,0 & 0,784 & $4,005 \cdot 10^{-5}$ & 52,0 & 2,02 & 0,595 & 0,210 \\
\hline$p H c 2$ & $\exp$ & $2.791 .10^{-2}$ & $5,772.10^{-2}$ & 123,6 & 0,971 & $3,928.10^{-6}$ & 51,6 & $6,90 \cdot 10^{-1}$ & 0,856 & 0,329 \\
\hline $\mathrm{DpH} 2$ & gau & $1,359 \cdot 10^{-2}$ & $3,108 \cdot 10^{-2}$ & 465,9 & 0,968 & $1,403 \cdot 10^{-5}$ & 56,3 & $-6,00.10^{-1}$ & 0,940 & 0,414 \\
\hline \multicolumn{11}{|c|}{$\gamma(h)$ simples do solo 0,80-1,00 m } \\
\hline$\# p H a 3$ & gau & $5,720.10^{-2}$ & $8,939 \cdot 10^{-2}$ & 98,3 & 0,992 & $3,426.10^{-6}$ & 36,0 & 0,00 & 0,932 & 0,383 \\
\hline pHk3 & epp & $2,362.10^{-1}$ & $2,362.10^{-1}$ & - & - & - & - & - & - & - \\
\hline$\# p H c 3$ & gau & $1,300.10^{-1}$ & $1,745.10^{-1}$ & 75,0 & 0,898 & $8,206.10^{-5}$ & 25,5 & 0,235 & $1,00.10^{-2}$ & 0,879 \\
\hline $\mathrm{DpH} 3$ & gau & $4,710.10^{-2}$ & $7,900.10^{-2}$ & 93,1 & 0,939 & $2,092 \cdot 10^{-5}$ & 40,4 & 0,409 & 0,000 & 0,999 \\
\hline
\end{tabular}

(a) TCH refere-se à produtividade de colmos por hectare; ATR refere-se aos açúcares totais recuperáveis; pHa, pHk, pHc e DpH são respectivamente atributos da reação do solo nas respectivas camadas (1: 0 - 0,20 m; 2: 0,20 - 0,40 m; 3: 0,80 - 1,00 m); \# trabalhado com o resíduo do atributo; ${ }^{(b)}$ gau = gaussiano, exp = exponencial, esf = esférico; epp = efeito pepita puro; (c) $\mathrm{SQR}=\mathrm{Soma}$ dos Quadrados dos Resíduos; (d) ADE = Avaliador da Dependência Espacial. 
Quadro 4. Parâmetros dos semivariogramas cruzados, ajustados entre os atributos da produtividade da cana-de-açúcar e do pH de um Argissolo Vermelho distrófico.

\begin{tabular}{|c|c|c|c|c|c|c|c|c|c|c|}
\hline \multirow{3}{*}{ Atributos $^{(a)}$} & \multicolumn{10}{|l|}{$\begin{array}{l}\text { Parâmetros } \\
\text { do ajuste }\end{array}$} \\
\hline & \multirow{2}{*}{ Modelo $^{(\mathbf{b})}$} & \multirow{2}{*}{$\mathrm{C}_{\mathrm{o}}$} & \multirow{2}{*}{$\mathrm{C}_{\mathrm{o}}+\mathrm{C}$} & \multirow{2}{*}{$\begin{array}{l}A_{0} \\
(\mathbf{m})\end{array}$} & \multirow{2}{*}{$\mathbf{r}^{2}$} & \multirow{2}{*}{$\mathbf{S Q R}^{(\mathrm{c})}$} & \multirow{2}{*}{$\begin{array}{c}\mathrm{ADE}^{(\mathrm{d})} \\
\%\end{array}$} & \multicolumn{3}{|c|}{ validação cruzada } \\
\hline & & & & & & & & $\mathbf{a}$ & B & $\mathbf{R}$ \\
\hline \multicolumn{11}{|c|}{$\gamma(h)$ cruzado TCH vs ATR } \\
\hline$\# T C H=f(A T R)$ & gau & $-1,000.10^{-1}$ & $-5,970 \cdot 10^{-1}$ & 248,2 & 0,795 & $1,250.10^{1}$ & 99,8 & $-8,02$ & 0,500 & 0,322 \\
\hline \multicolumn{11}{|c|}{$\gamma(h)$ cruzado TCH vs Atributos do solo } \\
\hline$\# T C H=f(p H k 2)$ & esf & $1,000.10^{-3}$ & $9,540.10^{-1}$ & 268,2 & 0,673 & $3,490.10^{-1}$ & 99,9 & $-8,62$ & 0,458 & 0,291 \\
\hline$\# T C H=f(p H c 2)$ & gau & $1,000.10^{-3}$ & $6,310.10^{-1}$ & 247,7 & 0,827 & $1,250.10^{-1}$ & 99,8 & $-7,36$ & 0,511 & 0,327 \\
\hline$\# T C H=f(D p H 1)$ & gau & $4,500.10^{-2}$ & $5,610.10^{-1}$ & 244,5 & 0,615 & $1,590.10^{-1}$ & 92,0 & $-7,62$ & 0,508 & 0,321 \\
\hline$\# T C H=f(D p H 2)$ & esf & $2,040.10^{-1}$ & $9,340.10^{-1}$ & 280,0 & 0,492 & $3,700.10^{-1}$ & 78,2 & $-6,46$ & 0,585 & 0,369 \\
\hline$\# T C H=f(D p H 3)$ & gau & $6,000 \cdot 10^{-3}$ & 1,177 & 148,2 & 0,799 & $2,280.10^{-1}$ & 99,5 & $-6,99$ & 0,573 & 0,369 \\
\hline \multicolumn{11}{|c|}{$\gamma(h)$ cruzado ATR vs Atributo do solo } \\
\hline$A T R=f(p H k 2)$ & gau & $-4,770.10^{-1}$ & $-1,393$ & 303,1 & 0,847 & $1,180.10^{-1}$ & 65,8 & $5,44.10^{1}$ & 0,605 & 0,460 \\
\hline$A T R=f(p H c 2)$ & esf & $-3,610.10^{-1}$ & $-8,690 \cdot 10^{-1}$ & 274,3 & 0,825 & $4,340.10^{-2}$ & 58,5 & $5,30.10^{1}$ & 0,615 & 0,471 \\
\hline$A T R=f(\# p H c 3)$ & esf & $-9,600.10^{-2}$ & $-1,366$ & 127,5 & 0,406 & $7,120.10^{-1}$ & 93,0 & $4,89.10^{1}$ & 0,646 & 0,493 \\
\hline$A T R=f(p H a 1)$ & esf & $-8,000.10^{-2}$ & $-6,100 \cdot 10^{-1}$ & 177,1 & 0,842 & $2,590 \cdot 10^{-2}$ & 86,9 & $5,11.10^{1}$ & 0,629 & 0,480 \\
\hline$A T R=f(p H a 2)$ & gau & $-2,000.10^{-1}$ & $-6,910 \cdot 10^{-1}$ & 240,0 & 0,493 & $1,820.10^{-1}$ & 71,1 & $5,04.10^{1}$ & 0,636 & 0,496 \\
\hline$A T R=f(\# p H a 3)$ & esf & $-2,650.10^{-1}$ & $-1,270$ & 177,0 & 0,681 & $2,210.10^{-1}$ & 79,1 & $4,78.10^{1}$ & 0,655 & 0,506 \\
\hline$A T R=f(D p H 3)$ & gau & $-1,000.10^{-4}$ & $-2,762 \cdot 10^{-1}$ & 102,5 & 0,854 & $7,642 \cdot 10^{-3}$ & 85,4 & $5,35 \cdot 10^{1}$ & 0,612 & 0,469 \\
\hline
\end{tabular}

(a) TCH refere-se à produtividade de colmos por hectare; ATR refere-se aos açúcares totais recuperáveis; $\mathrm{pHa}$, pHk, $\mathrm{pHc}$ e DpH são respectivamente atributos da reação do solo nas respectivas camadas (1: 0 - 0,20 $\mathrm{m} ; 2: 0,20$ - 0,40 m; 3: 0,80 - 1,00 m); \# trabalhado com o resíduo do atributo; (b) gau = gaussiano, esf = esférico; (c) $\mathrm{SQR}=$ Soma dos Quadrados dos Resíduos; ${ }^{\text {(d) }} \mathrm{ADE}=$ Avaliador da Dependência Espacial.

Já nos parâmetros semivariográficos cruzados entre os atributos da planta vs solo (Quadro 4), observou-se bons ajustes com alcances variando entre $102,5 \mathrm{~m}$ a $302,1 \mathrm{~m}$. Assim, do ponto de vista prático agronômico, o destaque, indicado pelo coeficiente de determinação $\left(\mathrm{r}^{2}\right)$, foi para: 1) $\# \mathrm{TCH}=f(\mathrm{pHc} 2)(0,827)$, que se deu de modo direto entre causa e efeito, com alcance de 168,2 $\mathrm{m} ; 2) \mathrm{TCH}=f(\mathrm{DpH} 3)$ também de modo direto, com ajuste gaussiano, alcance de 148,2 m e coeficiente de determinação $(0,799)$; e, 3$)$ para $\mathrm{ATR}=f(\mathrm{DpH} 3)$ que apresentou-se de modo indireto, com ajuste gaussiano, alcance de $102,5 \mathrm{~m}$, com um $\left(\mathrm{r}^{2}\right)$ de 0,854 e elevado ADE.

Nas Figuras 1 e 2, estão contidos os ajustes semivariográficos cruzados (a) e os mapas de cokrigagens (b) de maior interesse agronômico e de melhor desempenho. Portanto, a cokrigagem $\# \mathrm{TCH}=f(\mathrm{pHc} 2)$ (Figura 1a,b) permitiu constatar que no presente trabalho a variabilidade espacial da TCH pode ser determinada por meio do $\mathrm{pH}$ em $\mathrm{CaCl} 2$ na camada de $0,20-0,40 \mathrm{~m}$ com uma precisão estimativa de 82,7\% (Quadro 4). Portanto, para área estudada, nos locais amostrados onde o $\mathrm{pHc} 2$ apresentar valores entre 4,5-4,7, a TCH esperada da cana-de-açúcar, RB 855453, será de 37-74 t ha ${ }^{-1}$. Por outro lado, naqueles sítios onde o $\mathrm{pHc} 2$ estiver entre 4,8-5,0 a TCH estará entre 87-124 tha ${ }^{-1}$.

$\mathrm{O} \mathrm{DpH} 3$ não denotou resposta favorável, do ponto de vista do manejo agrícola com $\mathrm{TCH}$, porém em relação ao ATR (Quadro 4; Figura 2) os resultados indicaram que o incremento da CTC na camada de 0,80-1,00 m proporcionou melhor qualidade do produto industrializável, ou seja, nos sítios onde o Delta $\mathrm{pH}$ apresentou maiores valores negativos, os valores de ATR foram substancialmente maiores e vice versa. Sendo tal fato, de grande importância agronômica uma vez que o ATR determina o preço da cana pago pela indústria.

A análise da dependência espacial simples e cruzada (Quadros 3 e 4; Figuras 1 e 2) permitiu concluir que, considerando a produtividade da cana-de-açúcar (TCH) tendo variado de 37-74 tha-1 
a)

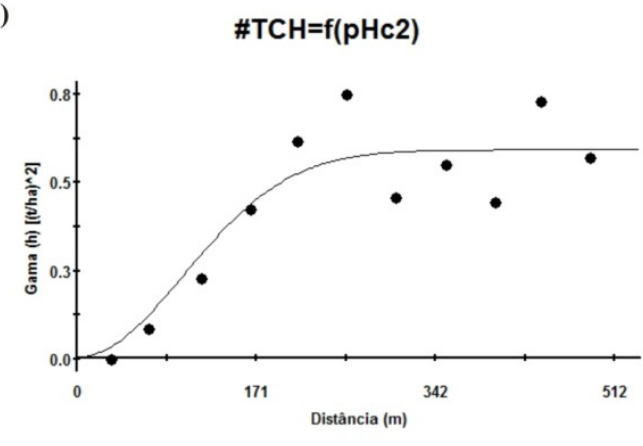

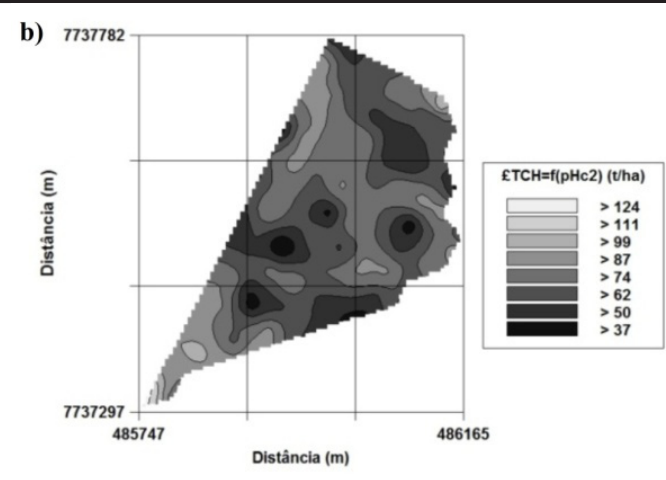

Figura 1. Semivariograma e mapa de cokrigagem entre a produtividade da cana-de-açúcar e o $\mathrm{pH}$ de um Argissolo Vermelho distrófico.
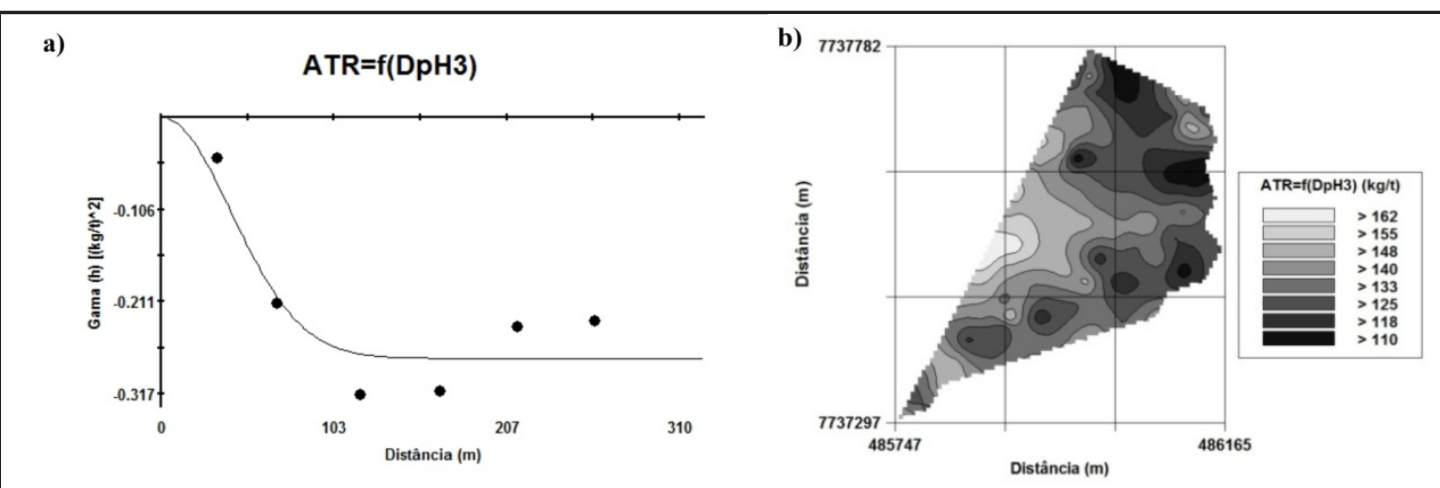

Figura 2. Semivariograma e mapa de cokrigagem entre o ATR da cana-de-açúcar e o DpH de um Argissolo Vermelho distrófico.

(menores valores) a 74-87 tha - $^{-1}$ maiores valores), do mesmo modo que o componente tecnológico (ATR) que variou de $110-133 \mathrm{~kg} \mathrm{t}^{-1}$ (menores valores) a $140-162 \mathrm{~kg} \mathrm{t}^{-1}$ (maiores valores), e ambos tendo se correlacionado espacialmente com os atributos do solo, variando segundo padrões apresentados pela heterogeneidade espacial dos atributos do $\mathrm{pH}$, há uma real necessidade em trabalhar com áreas e perfis específicos de manejo a fim de reduzir a heterogeneidade dos sítios localizados de interação com os menores valores de TCH e/ou ATR, e otimizar assim a produção agrícola seja pelo incremento da produtividade ou pelo ganho em qualidade do produto industrializável final.

\section{CONCLUSÕES}

- A correlação linear entre a produtividade da cana-de-açúcar com o $\mathrm{pH}$ do solo é significativa na profundidade de 0,80-1,00 m (pHk3, pHc3);
- Do ponto de vista espacial, há apreciável resposta entre a produtividade da cana-deaçúcar com o pHc2 do solo, permitindo inferir que a adequação química das áreas específicas de maior acidez proporcionará incremento na produtividade da cultura;

- O Delta pH do solo na camada de 0,80-1,00 $\mathrm{m}$ apresenta interação espacial com o ATR, indicando que uma melhor qualidade química naquela camada melhorou a qualidade final do produto industrializável, com ATR mais elevado.

\section{REFERÊNCIAS BIBLIOGRÁFICAS}

BERNER, P.G.M.; VIEIRA, S.R.; LIMA, E.; ANJOS, L.H.C. Variabilidade espacial de propriedades físicas e químicas de um Cambissolo sob dois sistemas de manejo de cana-de-açúcar. Revista Brasileira de Ciência do Solo, v.31, p.837-844, 2007.

\section{REVENG 120-130p.}




\section{COMPANHIA}

NACIONAL

DE

ABASTECIMENTO

(CONAB).

Acompanhamento de safra brasileira: cana-deaçúcar. Safra 2013/2014. Quarto levantamento. Brasília: Conab, 2014. 19p.

CAMPOS, M.C.C.; MARQUES JÚNIOR, J.; PEREIRA, G.T.; FREITAS, E.V.S. Dependência espacial de atributos químicos em área cultivada com cana-de-açúcar em Pereira Barreto, SP. Revista Ciência Agronômica, v.38, p.350-359, 2007.

CONSELHO DOS PRODUTORES DE CANADE-AÇÚCAR, AÇÚCAR E ÁLCOOL DO ESTADO DE SÃO PAULO (CONSECANA). Manual de instruções. Piracicaba, 2006. 116p.

CORÁ, J.E.; ARAUJO, A.V.; PEREIRA, G.T.; BERALDO, J.M.G. Variabilidade espacial de atributos do solo para adoção do sistema de agricultura de precisão na cultura de cana-deaçúcar. Revista Brasileira de Ciência do Solo, v.28, p.1013-1021, 2004.

CORÁ, J.E.; BERALDO, J.M.G. Variabilidade espacial de atributos do solo antes e após calagem e fosfatagem em doses variadas na cultura de canade-açúcar. Engenharia Agrícola, v.26, p.374-387, 2006.

DALCHIAVON F.C.; CARVALHO, M.P.; NOGUEIRA, D.C.; ROMANO, D.; ABRANTES, F.L.; ASSIS, J.T.; OLIVEIRA, M.S. Produtividade da soja e resistência mecânica à penetração do solo sob sistema plantio direto no cerrado brasileiro. Pesquisa Agropecuária Tropical, v.41, p.08-19, 2011.

EMBRAPA - Empresa Brasileira de Pesquisa Agropecuária. Manual de análises químicas do solo, plantas e fertilizantes. 2. ed. Brasília: 2009. $627 \mathrm{p}$.

EMBRAPA - Empresa Brasileira de Pesquisa Agropecuária. Sistema brasileiro de classificação de solos. 3. ed. Rio de Janeiro: 2013. 353p.

FAHL, J.I.; CAMARGO, M.B.P. de; PIZZINATTO,
M.A.; BETTI, J.A.; MELO, A.M.T.; DeMARIA, I.C.; FURLANI, A.M.C. (Eds.). Instrucoes agricolas para as principais culturas economicas. 6.ed.rev.atual. Campinas: IAC, 1998. 393p. (IAC. Boletim, 200).

FREDDI, O.S.; CARVALHO, M.P.; VERONESI JÚNIOR, V.; CARVALHO, G. Produtividade do milho relacionada com a resistência mecânica à penetração do solo sob preparo convencional. Engenharia Agrícola, v.26, p.113-121, 2006.

MARQUES JÚNIOR, J.; SOUZA, Z.M.; PEREIRA, G.T.; BARBIERI, D.M. Variabilidade espacial de matéria orgânica, $\mathrm{P}, \mathrm{K}$ e CTC de um latossolo cultivado com cana-de-açúcar por longo período. Revista de Biologia e Ciências da Terra, v.8, p.143-152, 2008.

MELLO, G.; BUENO, C.R.P.; PEREIRA, G.T. Variabilidade espacial das propriedades físicas e químicas do solo em áreas intensamente cultivadas. Revista Brasileira de Engenharia Agrícola Ambiental, v.10, p.294-305, 2006.

NOVAIS, R.F.; ALVAREZ, V.V.H.; BARROS, N.F.; FONTES, R.L.F.; CANTARUTTI, R.B.; NEVES, J.C.L. (Eds.) Fertilidade do solo. Viçosa: SBCS, 2007. 1017p.

RAIJ, B.V. Fertilidade do solo e adubação. Piracicaba: Ceres, 1991. 343p.

RAIJ, B.V.; CANTARELLA, H.; QUAGGIO, J.A.; FURLANI, A.M.C. Recomendações de adubação e calagem para o Estado de São Paulo. Campinas: Instituto Agronômico, 1997. 285p.

ROBERTSON, G.P. GS+: geostatistics for environmental sciences. 7.ed. Michigan, Plainwell: Gamma Desing Software, 2004. 159p.

SCHLOTZHAVER, S.D.; LITTELL, R.C. SAS system for elementary statical analysis. 2.ed. Cary: SAS, 1997. 441p.

SEGATO, S.V.; MATTIUZ, C.F.M.; MOZAMBANI, A.E. Aspectos fenológicos da 
cana-de-açúcar. In: SEGATO, S.V.; PINTO, A.S.; JENDIROBA, E. (Org.). Atualização em produção de cana-de-açúcar. Piracicaba: Livroceres, 2006. p.19-36.

SHAPIRO, S.S.; WILK, M.B. An analysys of variance test for normality: complete samples. Biometrika, v.52, n.3/4, p.591-611, 1965.

SOUZA, Z.M.; BARBIERI, D.M.; MARQUES JÚNIOR, J.; PEREIRA, G.T. \& CAMPOS, M.C.C. Influência da variabilidade espacial de atributos químicos de um Latossolo na aplicação de insumos para cultura de cana-de-açúcar. Ciência Agrícola, v.31, p.371-377, 2007.

SOUZA, Z.M.; CERRI，D.G.P.; COLET, M.J.; RODRIGUES, L.H.A.; MAGALHÃES, P.S.G.; MANDONI, R.J.A. Análise dos atributos do solo e da produtividade da cultura de cana-de-açúcar com o uso da geoestatística e árvore de decisão. Ciência Rural, Santa Maria, v.40, n.4, p.840-847, 2010.

SOUZA, Z.M.; CERRI, D.G.P.; MAGALHÃES, P.G.; CAMPOS, M.C.C. Correlação dos atributos físicos e químicos do solo com a produtividade de cana-de-açúcar. Revista de Biologia e Ciências da Terra, Campina Grande, v.8, n.2, p.183-190, 2008.

SOUZA, Z.M.; MARQUES JÚNIOR, J.; PEREIRA, G.T.; MOREIRA, L.F. Variabilidade espacial do $\mathrm{pH}, \mathrm{Ca}, \mathrm{Mg}$ e V\% do solo em diferentes formas do relevo sob cultivo de cana-de-açúcar. Ciência Rural, v.34, p.1763-1771, 2004.

VIEIRA, S.R. Variabilidade espacial de argila, silte e atributos químicos em uma parcela experimental de um Latossolo Roxo de Campinas (SP). Bragantia, v.56, p.181-190, 1997. 